\title{
Gezeitenströmung und Nahrungsfiltration bei Ophiuren und Crinoiden
}

\author{
Dietrich B. E. Magnus \\ Zoologisches Institut der Tecbnischen Hochschule Darmstadt
}

\begin{abstract}
Tidal currents and food filtration in ophiurids and crinoids. At different seasons, long-term field observations, experiments and measurements were conducted on Opbiocoma scolopendrina and Heterometra savignyi in order to investigate their food uptake mechanism and its relation to environmental factors. O. scolopendrina lives in the tidal zone. It obtains food in three different ways: in quiet waters it collects or plucks food from the substratum; in running waters it filters, and while the tide is coming in, it removes (during the day time) dry particles from the water surface. In the filtration process, the ambulacral feet line up and form filtrating combs. This filtering mechanism works effectively, however, only in currents having a significant speed and containing larger suspended particles. It is suggested that Opbiothrix quinqueriaculata and O. fragilis also obtain their floating food exclusively by filtration from relatively fast currents rich in organic particles, rather than by collecting sinking particles as has often been assumed. Both species live in greater depths at the sea bottom, where they occassionally occur in great numbers. They lie on the floor, sometimes on their backs, and usually with their arms up. H. savignyi lives in shallow water areas having weak currents and feeds exclusively by filtration of fine, mostly living, suspended material. The animals arrange their 20 arms to form a shallow filtration fan and turn their aboral regions towards the current. The tentacles, stiffly stretched and between the pinnulae, form a fine filter net. Contrary to previously held opinions, these results suggest that the other crinoid species also obtain their food exclusively or predominantly by filtration from weak currents; it is not the catching funnel formed by the oral sides, but a shallow filter fan formed by the aboral arm surfaces that points toward the current.
\end{abstract}

\section{EINLEITUNG}

Untersuchungen über den Nahrungserwerb von Ophiuren und Crinoiden durch direktes Beobachten des Verhaltens, experimentelles Eingreifen und Messen der ökologischen Faktoren am natürlichen Standort über einen längeren Zeitraum sind bisher kaum durchgeführt worden. Ich wählte dazu je eine Art aus diesen beiden Echinodermengruppen, nämlich den Schlangenstern Opbiocoma scolopendrina (Lamarck) und den Federstern. Heterometra savignyi (J. Mül.LER), die beide im Roten Meer häufig sind und an leicht zugänglichen Stellen angetroffen werden können (MAGNus $1963 a$, b). Die Untersuchungen an $H$. savignyi sind schwimmtauchend vorgenommen worden.

Als Arbeitsplätze dienten im März und April 1961 die Hafenbucht von Suakin (H. savignyi), im Mai 1961 ein Küstenstreifen bei Kosseir (O. scolopendrina) und im 
September und Oktober 1962 die Gezeitenzone und Flachwassergebiete nahe der Meeresbiologischen Station der Universität Kairo in Al Ghardaqa (H. savignyi und O. scolopendrina).

\section{OPHIOCOMA SCOLOPENDRINA}

\section{Biotop}

Dieser Schlangenstern besiedelt poröse Hartböden mit teilweiser dünner Sedimentdecke in tieferen und mittleren Lagen der Gezeitenzone an brandungsgeschützten Küstenabschnitten, etwa von der MNW- bis fast zur MW-Linie hinauf. Bevorzugt werden zwar ständig wasserbedeckte Habitate in weiten flachen Mulden oder kleineren Tümpeln auf der Gezeitenplatte oder im Übergangsgebiet zu Saumriffen, doch nicht unbeträchtliche Teile der Populationen bewohnen auch Stellen, die bei jeder Tide trocken fallen, und zwar in gleicher Individuendichte. In der Regel handelt es sich um sehr große Populationen mit einer Dichte von $50 / \mathrm{m}^{2}$. Jedes Tier besitzt seine eigene Wohnhöhle, die es über Wochen hindurch nicht aufgibt und gegen Artgenossen und andere Biotopangehörige auf Berührungsreize hin durch Stoßen und Schieben mit den aboralen Armflächen verteidigt. Während der Nahrungsaufnahme verbleiben am Tage ein Arm und meist auch die Körperscheibe im Versteck, nachts mindestens eine Armspitze (Abb. 1).

\section{Aktivitätsphasen}

Die Aktivitätsphasen der Tiere werden von den Tiden- und Windverhältnissen sowie der jeweiligen Wohnplatzstruktur bestimmt. Je nach Zustand der Wasserbewegung und dem Nahrungsangebot am Einzelwohnplatz können Form, Zeit und Dauer des Nahrungserwerbs selbst auf kleinstem Raum von Tier zu Tier sehr unterschiedlich sein. Auch die Zahl der jeweils daran beteiligten Arme ist verschieden. Der Tidenhub beträgt an den Untersuchungsplätzen höchstens etwa $90 \mathrm{~cm}$. Die Überflutungszeit verbringen die gegen Wellen sehr empfindlichen Tiere inaktiv und in ihre dann meist mit Sand zugeschwemmten Schlupfwinkel zurückgezogen. Nur unter genügend tiefem Wasser, wo die Wellenbewegungen sie nicht mehr erreichen, z. B. in Mulden auf breiten Gezeitenplatten und bei windstillem Wetter, sind sie gelegentlich auch bei Flut aktiv. Daher findet der Nahrungserwerb fast ausschließlich bei Niedrigwasser in den dann abgeschlossenen und wellenfreien Tümpeln statt und auf den trocken fallenden Habitaten bei auf- oder ablaufendem Wasser, solange der Wasserstand über den Wohnhöhlen so niedrig ist (oft nur wenige $\mathrm{cm}$ ), daß sich keine Wellen ausbilden können.

\section{Nabrungserwerb}

Zur Nahrungsaufnahme aktiviert werden die Tiere durch Geschmacksreize, die das strömende Wasser heranführt oder die sich im stehenden Wasser ausbreiten (im 
Freilandversuch z. B. Fleisch- oder Pflanzenpreßsaft). Auf diese Reize erfolgen suchende schlängelnde Bewegungen der Arme, deren Spitzen leicht erhoben werden. Der Zustand der Wasserbewegung löst als mechanischer Reiz die spezifische Aufnahmeform aus. Eine Geschmacksprüfung durch die Ambulacralfüßchen entscheidet über die Aufnahme. Der Transport des Aufgenommenen in den Magen erfolgt mit einer durchschnittlichen

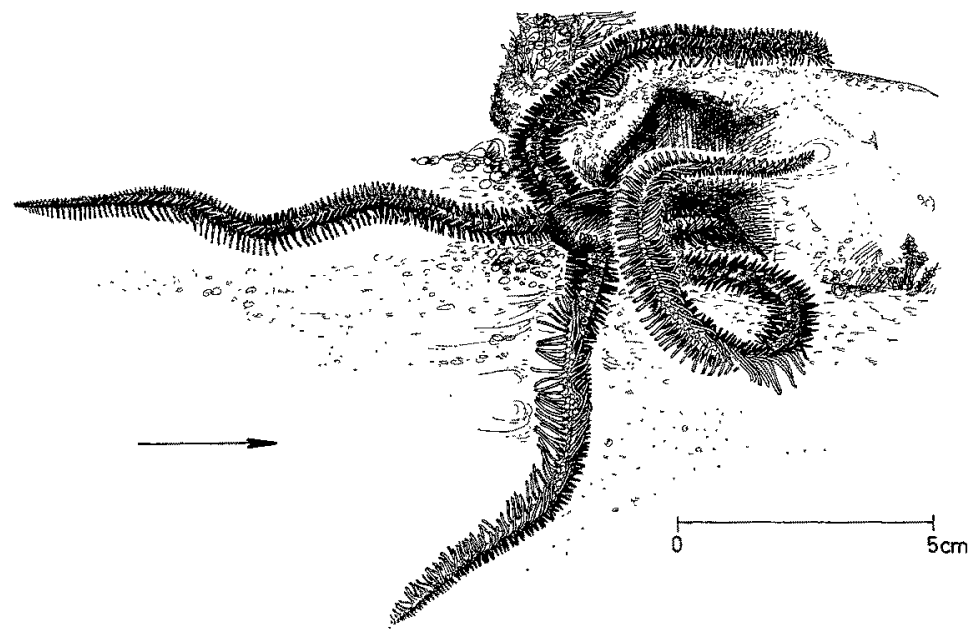

Abb. 1: Opbiocoma scolopendrina. Armhaltungen beim Nahrungserwerb in strömendem Wasser. Ein Arm in der Wohnhöhle, ein Arm bei der Nahrungsaufnahme von der Bodenoberfläche, drei Arme in Filtrationsstellung. $\rightarrow=$ Strömungsrichtung

Geschwindigkeit von $6,9 \mathrm{~cm} / \mathrm{min}$ zwischen den beiden Füßchenreihen an der oralen Armseite durch Weitergabe von Füßchen zu Füßchen unter gleichzeitigem Einhuillen in Drüsensekret. Gefressen werden lebende und abgestorbene tierische und pflanzliche Organismen bzw. Teile davon, wobei die Pflanzennahrung bei weitem überwiegt.

Bei O. scolopendrina können drei verschiedene Formen der Nahrungsaufnahme unterschieden werden:

1. Absammeln oder Abrupfen von der Bodenoberfläche. Bei dieser im unbewegten oder sehr schwach strömenden Wasser häufigsten Ernährungsweise tasten die Arme langsam über den Boden hin, bis sie Kontakt mit freßbaren Partikeln gefunden haben, und sammeln sie mit den Füßchen auf (Abb. 1). Algenbewuchs der Wohnhöhlenumgebung rupfen die Tiere $a b$, indem sie Teilchen davon mit den Füßchen bzw. deren Drüsensekret festhalten und dann den Arm ruckartig einkrümmen und zurüdkziehen.

2. Filtrieren. Im strömenden Wasser werden die Arme in ganzer Länge oder nur an den Enden vom Boden abgehoben und in den Strom gehalten. Die Füßchen sind dabei steif ausgestreckt und bilden zwei grobe Filterkämme, die sich in einem Winkel von etwa $90^{\circ}$ gegenüberstehen. Die Bewegungen der filtrierenden Arme eines Individuums sind nicht koordiniert, jeder Arm handelt unabhängig (Abb. 1). Zwar werden bei sehr niedrigem Wasserstand die filtrierenden Arme häufig quer zur Strömungsrichtung gehalten und dabei die Oralseiten zum Boden oder schräg gegen die 
Strömung gerichtet, bei höherem Wasser jedoch zeigt die Stellung der Arme in der Regel keine so deutliche Ausrichtung zur Strömung. Sie können dann schraubig verdreht sein und ändern auch ihre Einstellung oft. Aber stets bleiben die Füßchen gerade und weit ausgestreckt (Abb. 2a). Die mit der Strömung an die Füßchenkämme geführten Partikel werden von deren Drüsenschleim festgehalten und nach einer Geschmacks-

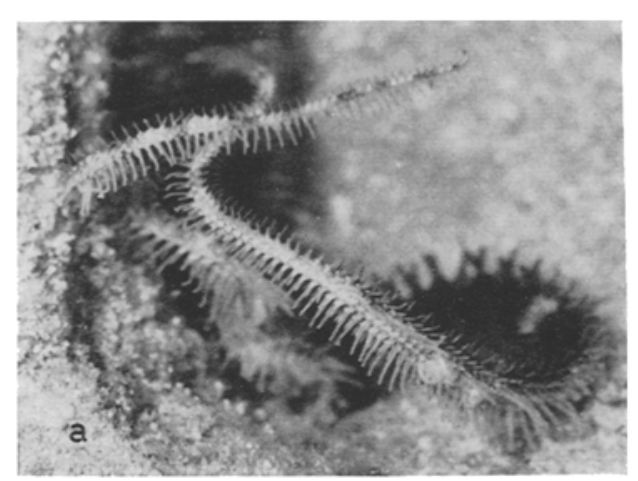

Abb. 2a

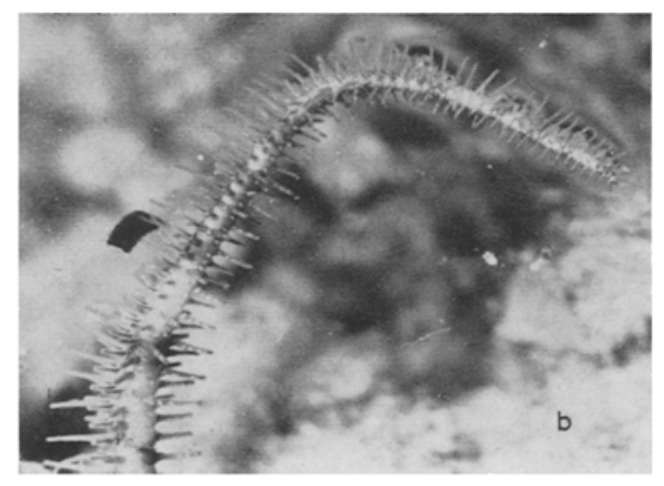

Abb. 2b

Abb. 2: Opbiocoma scolopendrina. Filtrationsstellung der Ambulacralfüßchen. - a. Transport in Füßchen-Drüsensekret gehüllter Nahrungspartikel zwischen den Füßchen-Filterkämmen der oralen Armseiten. - b. Aufnahme eines Seegras-Blattstïckes aus der Strömung

prüfung entweder wieder abgestoßen oder aufgenommen (Abb. 2a, b). Dieser Filterapparat aus den Ambulacralfüßchen ist sehr grob und die Filtration bei stärkerer Strömung am wirksamsten, wenn die Arme tiefgehalten werden und dann den dicht über den Boden treibenden gröberen Detritus abseihen können. Es werden dabei auch größere Partikel, z. B. Sargassum-Büschel, eingefangen und in den Magen aufgenommen.

Wenn die Tiere an ihrem Wohnplatz dazu Gelegenheit haben, erklettern sie, vor allem nachts und bei schwacher Strömung, zum Filtrieren oft erhöhte Stellen, wie Felsvorsprünge, Algen oder Seegrasblätter, jedoch nur soweit, daß noch eine Armspitze 
im Versteck bleibt. Hierbei fangen sie auch allerlei Kleintiere, wie z. B. Krebse und kleine Polychaeten, die aktiv zufällig gegen die wie Leimruten wirkenden Füßßhen schwimmen.

3. Abweiden des Staubfilms von der Wasseroberfläche (Magnus 1963a). Dieses spezialisiert anmutende und von den beiden anderen Ernährungsweisen auffällig verschiedene Verhalten kann nur von solchen Tieren, deren Versteck bei Ebbe trockengefallen war, und nur am Tage ausgeführt werden, weil nur dann das auflaufende Wasser eine dünne, sonnengetrocknete, hauptsächlich aus Diatomeen und anderen Algen bestehende Staubschicht von der Ebbezone abheben und mit sich führen kann. Bei der Überflutung kommen die aus den Höhlen herausgestreckten Schlangensternarme mit der Wasseroberfläche und deren Staubfilm in Berührung. Auf diesen Reiz hin drehen die Arme sich herum, legen sich mit der Oralseite von unten her an die Wasseroberfläche, durchbrechen diese und führen an der Wasseroberfläche schnelle schlängelnde Pendelbewegungen aus, wobei sich zwei benachbarte Arme genau spiegelbildlich verhalten. Da sich stets die Spitzen zweier gegeneinander schwingender Arme zuerst treffen, kann der zwischen ihnen eingefangene Staubfilm nicht entweichen und wird zusammen- und gegen die Füßchen gedrückt. Diese heben ihn aus dem Wasser auf die orale Armseite und schleimen und drücken ihn zu einer Nahrungswurst zusammen. Die Zeitdauer für ein einmaliges Zusammenschlagen und Auseinanderschwingen zweier Arme beträgt durchschnittlich nur 2,25 Sek. Allerdings wird hierbei keine Auswahl zwischen brauchbaren und unbrauchbaren Partikeln vorgenommen, weil im natïrlichen „Staub“ die positiven Geschmadksreize überwiegen. Wenn experimentell das Reizverhältnis geändert wird, unterbleibt die Aufnahme (Magnus \& VöLKER in Vorbereitung).

\section{Diskussion (O. scolopendrina)}

Die Nahrungsaufnahme durch Filtration aus dem Gezeitenstrom ist bei O. scolopendrina nur eine von drei möglichen Formen des Nahrungserwerbs, wird meist nur relativ kurzfristig ausgeführt und hat hier sicher keine große Bedeutung. Bei anderen Schlangensternarten spielt die Filtration anscheinend eine bedeutend wichtigere Rolle oder ist unter Umständen die einzige Form des Nahrungserwerbs, wie es z. B. bei Ophiotbrix quinquemaculata und Ophiotbrix fragilis der Fall $\mathrm{zu}$ sein scheint, die beide in zum Teil sehr großen Mengen den Meeresboden in größeren Tiefen bewohnen (Vevers 1952, Riedl 1956, 1963, Czihak 1959, Czihak \& Zei 1960). Die mit automatischen Kameras aufgenommenen Bilder aus diesen Populationen zeigen, daß ein großer Teil der Tiere den Rücken dem Substrat zuwendet. Sowohl diese umgekehrt liegenden wie auch alle übrigen Individuen halten einzelne oder auch mehrere Arme frei ins Wasser ausgestreckt. Es kann sicher kein Zweifel darüber bestehen, daß sie Geschwebe filtrieren und vielleicht auch gelegentlich Leimrutenfang ausüben, wenn die Wasserströmung schwach ist. Bei einigen der von VEvERs (1952) veröffentlichten Bilder scheint sogar eine Ausrichtung der Arme vieler der dort in mehreren Schichten übereinander liegenden $O$. fragilis zur Strömungsrichtung angedeutet zu sein. Daß keine deutliche Strömungsausrichtung zu erkennen ist, dürfte seinen Grund darin haben, daß 
bei der Art des Filtrierens der Ophiuren eine bestimmte Armstellung keine große Rolle spielt, wie die Beobachtungen an O. scolopendrina erwiesen haben. Aus der Tatsache, daß trotz dieser unvollkommenen Art der Filtration der Ophiuren offensichtlich genügend Nahrung für derartig viele und relativ große Tiere aus dem Strom herausgesiebt werden kann, muß auf stetige und kräftige Strömungen, die viel organisches Geschwebe dicht über den Grund treiben, geschlossen werden. Allerdings ist bisher über das Strömungsklima an solchen Standorten kaum etwas bekannt.

Man hat bis in die neueste Zeit die Vermutung geäußert, daß diese Schlangensterne, die mit dem Rücken am Boden liegen, sich von einem „Detritusregen“, der von oben käme, ernähren sollten. Diese Ansicht kann auf keinen Fall im wörtlichen Sinne zutreffen, denn man sieht auf den veröffentlichten Bildern neben den Schlangensternen Steine und andere Hartbodengebilde liegen, die bei einem stetigen und zur Ernährung dieser Tiermengen ausreichenden Regen organischen Materials von diesem oder zumindest von den Exkrementen der Schlangensterne längst zugedeckt sein müßten. Die Strömung bringt nicht nur die Nahrung, vermutlich in Form nahe am Grund treibenden Geschwebes, herbei, sondern schafft auch den Kot der Tiere fort.

Versuche im Aquarium mit $O$. scolopendrina zeigten, daß diese Tiere sich auf einen im strömungsfreien Wasser gebotenen Futterregen (in Seewasser aufgeweichtes "Piscidin"-Fischfutter) hin keineswegs auf den Rücken legten und auch nicht ihre Arme umdrehten, um die Oralseiten dem "Regen“ entgegenzuwenden. Sie nahmen das herab. regnende Futter in der im stehenden Wasser üblichen Weise nach seiner Sedimentation von der Bodenoberfläche auf. Man darf wohl daraus schließen, daß sich andere Schlangensterne am Meeresgrund in nicht strömendem Wasser genauso verhalten. Im unbewegten Wasser fehlt offensichtlich der zum Aufrichten der Arme erforderliche Auslösereiz, und die geringe Auftreffgeschwindigkeit des sedimentierenden Futters auf die Arme kann ihn nicht ersetzen. Im übrigen würde die Nahrung, die in einem angenommenen strömungsfreien Wasser nur oder vorwiegend durch Sedimentation an die Tiere herankäme, niemals ausreichen können, um diese großen Mengen von Schlangensternen zu ernähren.

\section{HETEROMETRA SAVIGNYI}

\section{Biotop}

Dieser im erwachsenen Zustand zwanzigarmige Federstern (Armlänge bis $25 \mathrm{~cm}$ ) bewohnt Seegraswiesen und Hartböden in brandungsgeschützten lagunenartigen Flachwassergebieten mit mäßigen Wasserströmungen zwischen Saumriffen oder in Hafenbuchten (Suakin). Sedimentgrund kann besiedelt werden, wenn er mit Hartbodenteilen durchsetzt ist, die den Tieren Halt bieten. Die Populationsdichte ist sehr unterschiedlich. Auf Seegraswiesen betrug sie höchstens bis $\mathrm{zu} 10 / \mathrm{m}^{2}$, meist jedoch bedeutend weniger. Die obere Grenze der Wohnhabitate gegen die Gezeitenzone hin liegt örtlich verschieden tief und fällt etwa mir der Tiefenlinie zusammen, von der ab keine Wellenbewegungen den Boden mehr erreichen, gegen die die Tiere sehr empfindlich sind. Im Hafenbecken von Suakin sitzen die Federsterne z. T. dicht unter der NW-Linie. 
Auf Seegras vor der M.B.-Station Ghardaqa findet man sie von etwa 1,50 m unter MW nach abwärts (MAGNus 1963b).

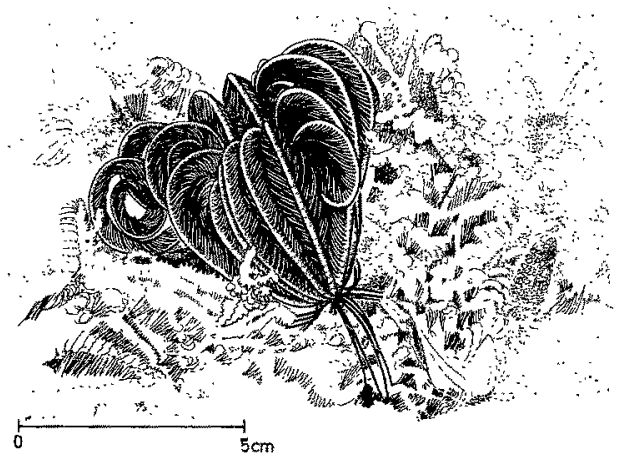

Abb. 3: Heterometra savignyi. Tages-Ruhestellung $z$ wischen Seegras in $2,50 \mathrm{~m}$ Tiefe. Nach Photographie

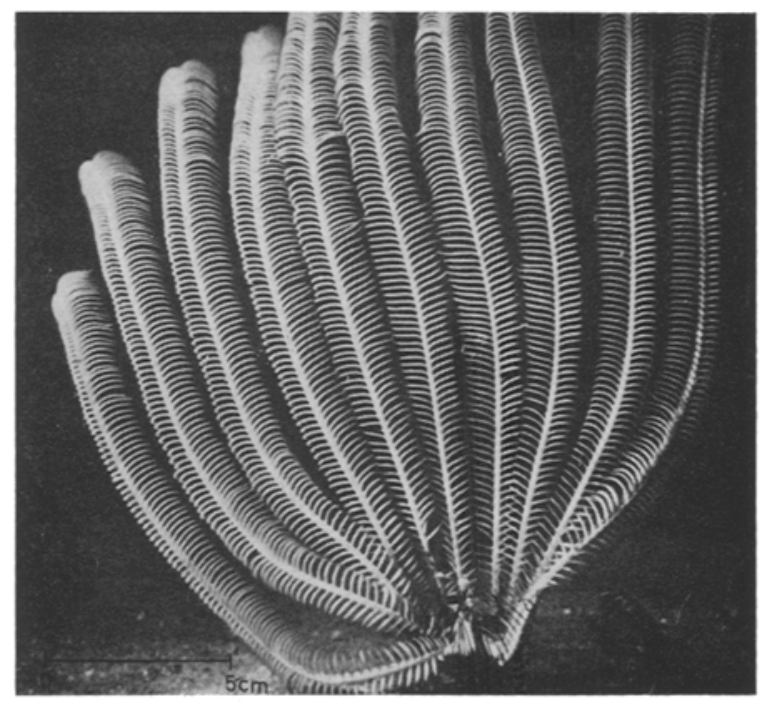

Abb. 4: Strömungsabgewandte, orale Armseiten des Filtrationsfächers eines 10armigen Jungtieres von Heterometra savignyi, nachts in $2 \mathrm{~m}$ Tiefe. Die seitlich stehenden Arme sind in der Längsachse um $180^{\circ}$ verdreht. Nach Farbphotographie

\section{Aktivitätsphasen}

Die Tiere sind nachtaktiv. Tagsüber sitzen sie, die Arme über dem Rumpf in engen Spiralen zusammengerollt, in schattigen Höhlen oder Spalten von Kaimauern, zwischen Seegras oder auch ganz frei, wenn keine Versteckmöglichkeiten vorhanden sind (Abb. 3 u. 8 ). Thre Aktivitätsperiode ist nicht überall allein auf die Dunkelzeit 
beschränkt, sondern kann, z. B. auf Seegraswohnplätzen, auch noch die Abend- und Morgenstunden mit geringen bis mäßigen Helligkeitswerten umfassen, anscheinend, weil die Tiere, je nach Belichtung der Tagesruheplätze, unterschiedlich adaptiert sind. Beginn und Ende des Nahrungsfangs sind daher standortbedingt unterschiedlich.

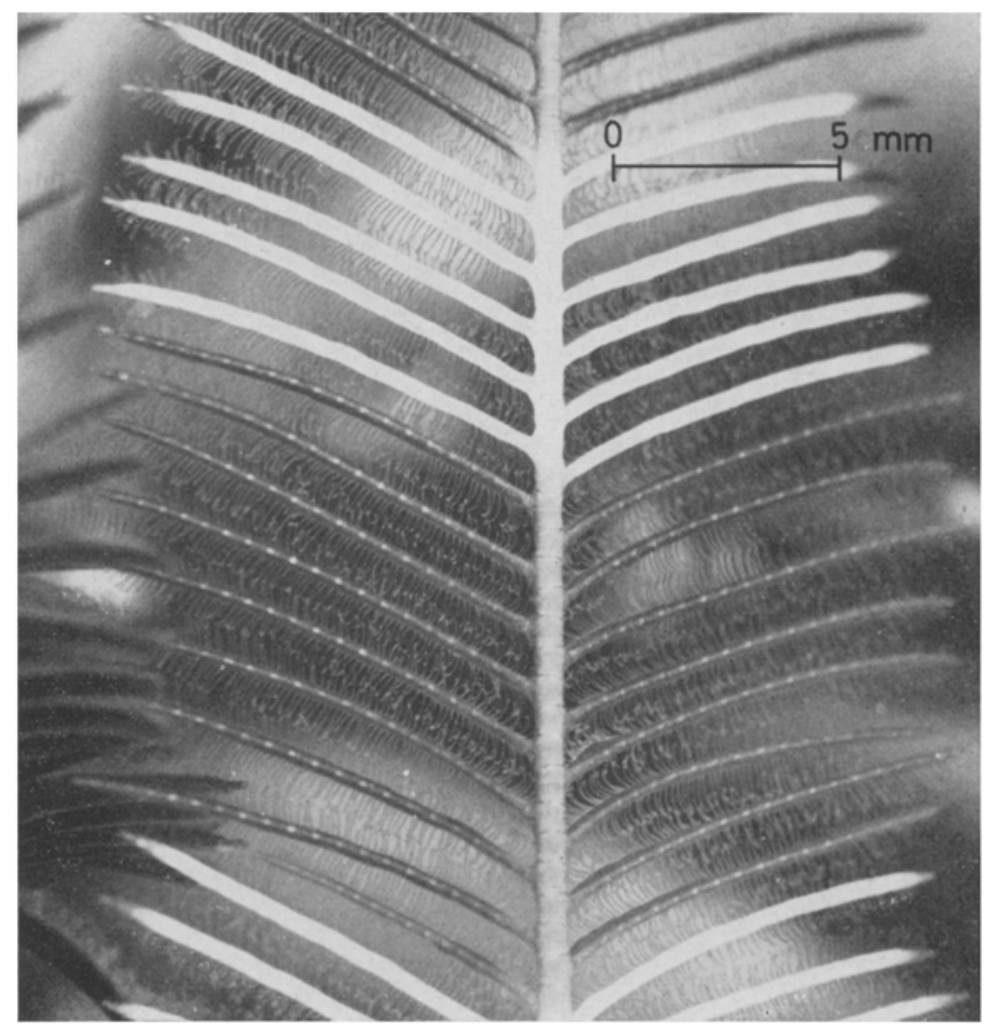

Abb. 5a

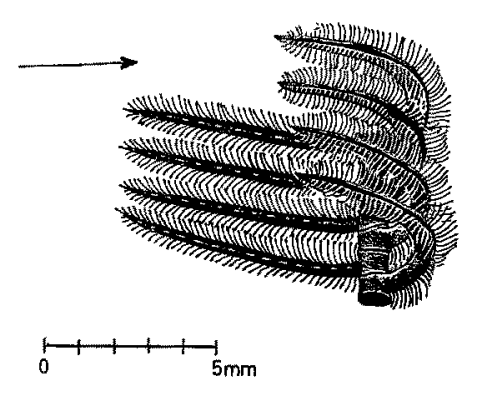

Abb. 5b

Abb. 5: Heterometra savignyi. Pinnulae in Filtrationsstellung. - a. Aborale Seite eines weiß gefleckten Armes. Die seitlich ausgestreckten Pinnula-Tentakel bilden ein feinmaschiges Filternetz. - b. Pinnulae, mit teilweise geöffneten Nahrungsrinnen, in typischer Filtrationsstellung zur aboralen Seite, gegen die Strömung, abgebogen. $\rightarrow=$ Strömungsrichtung. Nach Photographie 


\section{Nabrungserwerb}

Die Nahrungsaufnahme erfolgt offensichtlich ausschließlich durch Filtration aus der Strömung. Zum Nahrungsfang suchen die Tiere leicht erhöhte Plätze zu ersteigen, z. B. die Spitzen der Seegrasblätter oder Felsvorsprünge, und entrollen zunächst nur wenige, später alle Arme. Jeder Arm stellt sich so zur Strömungsrichtung ein, daß seine

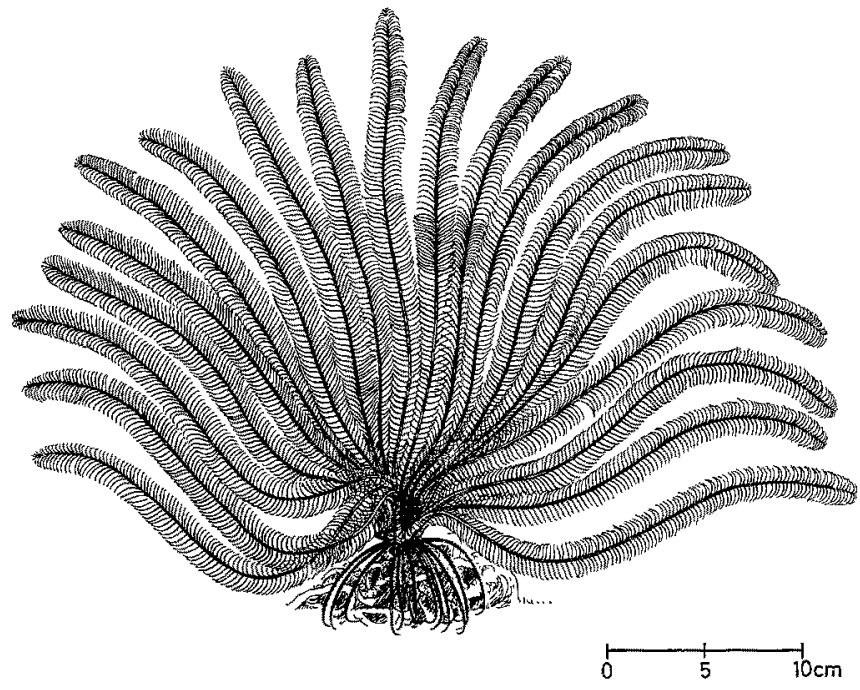

Abb. 6a

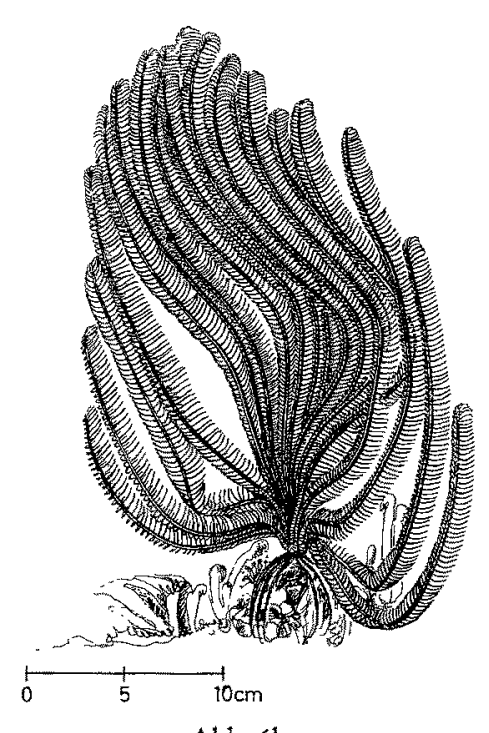

Abb. $6 \mathrm{~b}$

Abb. 6: Heterometra savignyi. Typischer Filtrationsfächer in schwacher Gezeitenströmung von etwa $3 \mathrm{~cm} / \mathrm{sec}$, nachts in $3 \mathrm{~m}$ Tiefe. - a. Orale, strömungsabgewandte Armseiten. - b. Filtrationsfächer, schräg von der Seite. Die Strömung kommt von rechts hinten. Nach Photographien 
aborale Seite möglichst voll gegen sie steht. Die Pinnulae, in Ruhehaltung auf die orale Armseite gelegt, stellen sich auf und biegen sich zur aboralen Seite herum, so daß sie in ihrer Gesamtheit auf jedem Arm ein gegen den Strom offenes, rinnenförmiges Gatter bilden (Abb. 4). Die kontraktilen Tentakel, die an den Hautsäumen der PinnulaNahrungsrinnen sitzen, strecken sich soweit zur Seite hinaus, daß sie an die Tentakel der benachbarten Pinnulae stoßen, und bilden in dieser Stellung ein sehr feinmaschiges Fangnetz (Abb. 5a, b). Während die Arme sich gegen die Strömung ausrichten, stellen sie sich nebeneinander in eine Ebene, und es entsteht daher schon bald aus den $20 \mathrm{Ar}-$ men ein einheitlicher flacher Filtrationsfächer (Abb. 4 u. 6a, b). Dazu müssen die an der strömungsabgewandten Körperseite entspringenden Arme bis zu $180^{\circ}$ um ihre Längsachse verdreht werden (Abb. 4). Wenn zwei Tiere dicht nebeneinander sitzen, bilden sie, da jeder Arm eine Stellung in ungestörter Strömung sucht, schließlich einen gemeinsamen, vierzigarmigen Fächer (Abb. 7).

Der Filtrationsfächer steht in der Regel aufrecht und quer zur Strömungsrichtung, die aboralen Armseiten ihr entgegengestellt. Durch steigenden Staudruck kann er niedergedrückt und schließlich aufgelöst werden. Doch scheinen sich die Tiere im allgemeinen nur an solchen Stellen aufzuhalten, wo vorwiegend gleichmäßige und schwache Strömungen herrschen. An den untersuchten Standorten lag die Strömungsgeschwindigkeit in der Regel etwa $z$ wischen $2-5 \mathrm{~cm} / \mathrm{sec}$, und die Fächer konnten sich gegen sie noch behaupten. Einem vorübergehenden stärkeren Strömungsdruck mußten sie nachgeben (Abb. 8). Im Untersuchungsgebiet bei Ghardaqa ist die Richtung der Gezeitenströmung von der jeweiligen Windrichtung abhängig, verläuft daher sowohl bei auf-wie bei ablaufendem Wasser in gleicher Richtung und ändert sich erst bei Anderung der Windrichtung. Bei einer solchen Richtungsänderung lösen sich die Fächer zunächst auf, und die Arme werden uneinheitlich nach oben und zur Seite gehalten, nie jedoch zu einem

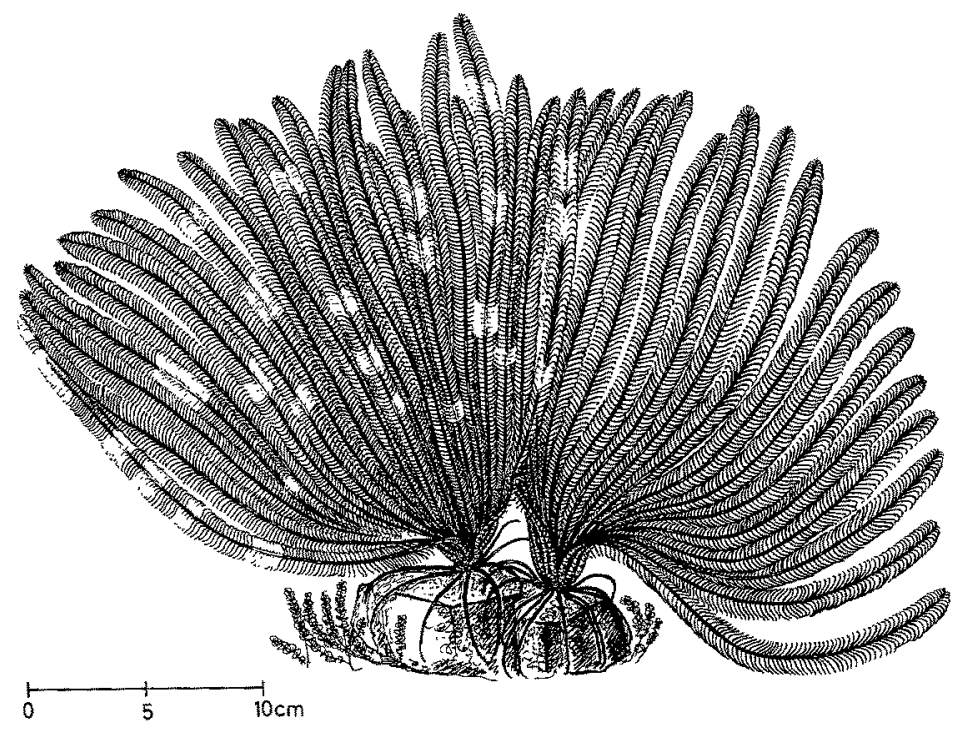

Abb. 7: Gemeinsamer, 40armiger Filtrationsfächer zweier nebeneinander sitzender Heterometra savignyi, nachts in $2,50 \mathrm{~m}$ Tiefe. Nach Photographie 
Kelch oder Trichter aufgestellt. Sowie dann das Wasser einheitlich in die neue Richtung fließt, richten sich die Arme wieder gleichmäßig gegen diese aus und bilden einen neuen Filtrationsfächer.

Die mit der Strömung herbeigeführten und als Nahrung geeigneten Planktonteilchen werden, wenn sie an die Tentakel stoßen, von diesen festgehalten und im Strömungsschatten an das Flimmerepithel der Pinnula-Nahrungsrinnen, die sich dazu öff-

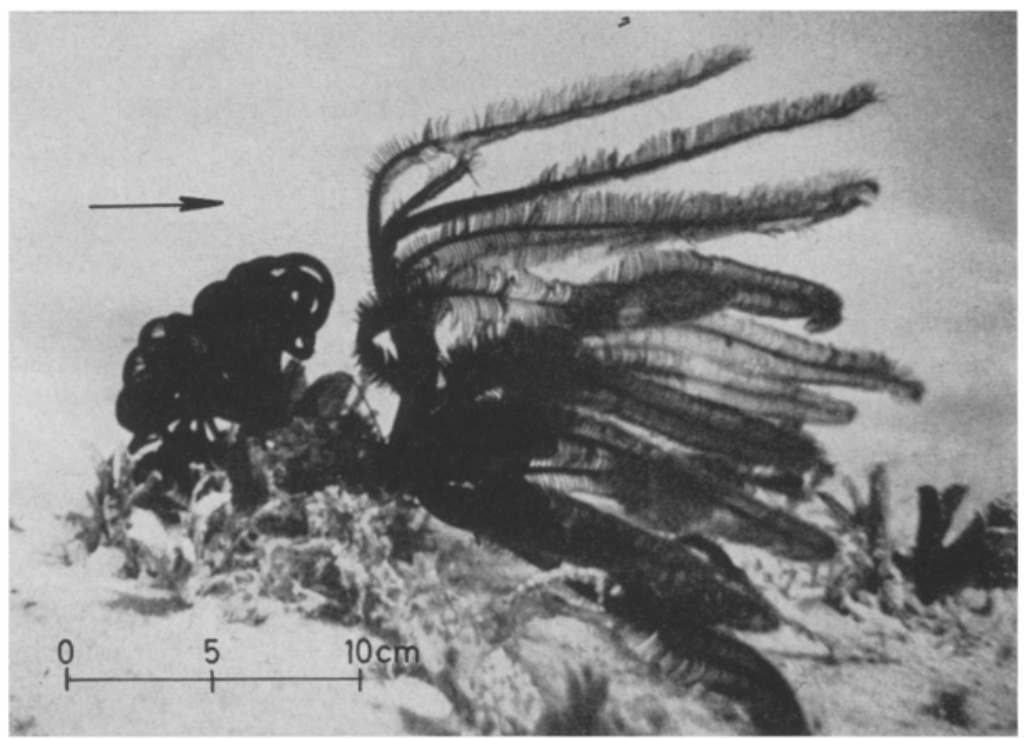

Abb. 8: Heterometra savignyi bei stärkerer Gezeitenströmung, die keine FiltrationsfächerBildung mehr zuläßt. Das linke Tier hat die Arme schon zur Tages-Ruhestellung eingerollt. Etwa 30 min nach Sonnenaufgang in $2 \mathrm{~m}$ Tiefe. $\rightarrow=$ Strömungsrichtung

nen, abgegeben. Die Nahrung besteht hauptsächlich aus lebenden kleinen und kleinsten Planktonorganismen: Bakterien, Diatomeen, Foraminiferen, Radiolarien, Kleinstcopepoden. Ungeeignete größere Teilchen werden dadurch aus dem Filternetz entlassen, daß die Pinnulae sich abwinkeln. Für ein erwachsenes 20 armiges Tier beträgt die Flächengröße des Filtrationsfächers mindestens etwa $500 \mathrm{~cm}^{2}$. Diesen Fächer passieren demnach bei einer Strömungsgeschwindigkeit von $2 \mathrm{~cm} / \mathrm{sec}$ annähernd mindestens $601 / \mathrm{min}$.

\section{Diskussion (O. savignyi)}

Diese Feststellungen über den Nahrungserwerb von $H$. savignyi stehen $z$. T. im Widerspruch zu unserer bisherigen Vorstellung über den Nahrungsfang der Crinoiden, die besagt, daß die Arme zu einer Art Trichter aufgestellt würden und dieser entweder einer schwachen Strömung entgegen oder aber in sehr ruhigem Wasser mehr oder weniger aufrecht gegen einen von oben herabschwebenden Nahrungsregen gerichtet würden, in jedem Fall also die oralen Armseiten die Nahrung abfangen sollten. 
Das staubfeine Geschwebe, welches den Crinoiden als Nahrung dient, sedimentiert teils überhaupt nicht (solange es lebend ist), teils viel zu langsam, als daß ein solcher Regen für die Tiere ausreichende Nahrung heranbringen könnte. Man muß wohl aus den Ergebnissen der Untersuchung an H. savignyi schließen, daß auch die anderen Crinoiden ihre Nahrung durch Filtration aus der Strömung abfangen, allerdings bei viel geringeren Fließgeschwindigkeiten als es die Ophiuren vermögen, und mit einem sehr viel wirksameren Filtrationsapparat als diese. Im Gegensatz zu den Ophiuren stellen sie jedoch die aboralen Armseiten der Strömung entgegen. Die oralen Seiten bleiben stets strömungsabgewandt. Die Tatsache, daß bei einer sehr langsamen Strömung von nur $2 \mathrm{~cm} / \mathrm{sec}$ annähernd etwa 400001 des in ihrem Flachwasserbiotop recht planktonreichen Wassers in einer Nacht den Filtrationsfächer einer $H$. savignyi passieren können, macht deutlich, daß diese Tiere trotz ihres feinmaschigen Filtrationsapparates verhungern müßten, wären sie allein auf absinkendes Material angewiesen. Die wenigen mir bisher bekannt gewordenen Bilder über andere Crinoiden-Arten an ihren natürlichen Standorten, zum Teil aus größeren Tiefen (EMERy 1952, MenzIes 1963), zeigen ebenfalls eine mehr oder weniger deutliche Filtrationsfächer-Stellung der Arme. Es scheint sich demnach um eine bei dieser Tiergruppe verbreitete Verhaltensweise zu handeln, vielleicht sogar um die normale Form des Nahrungserwerbes.

\section{ZUSAMMENFASSUNG}

1. Durch Untersuchungen an den natürlichen Standorten wurde versucht, den Nahrungserwerb und seine Beziehungen zu ökologischen Faktoren bei dem Schlangenstern Ophiocoma scolopendrina und dem Federstern Heterometra savignyi zu analysieren.

2. Die Aktivitätsphasen der ortsfesten, in der Gezeitenzone lebenden O. scolopendrina werden von den Tiden, den Wasserbewegungen und der Bodenstruktur am Wohnplatz bestimmt. Die wellenempfindlichen Tiere sind bei Hochwasser meist inaktiv. Der Nahrungserwerb findet in Gezeitentümpeln bei Niedrigwasser, an trocken fallenden Stellen nur solange das auf- oder ablaufende Wasser zu niedrig steht, als daß sich Wellen bilden können, statt.

3. Die Tiere sind zwar Allesfresser, doch überwiegt pflanzliche Nahrung.

4. Drei verschiedene Formen des Nahrungserwerbs sind zu unterscheiden: Absammeln oder -rupfen vom Boden (in unbewegtem Wasser), Filtrieren (in strömendem Wasser) und Abweiden des Staubfilms von der Wasseroberfläche (nur am Tage in auflaufendem Wasser).

5. Nahrungsaufnahme und -transport in den Magen erfolgen ausschließlich durch die Ambulacralfüßchen. Zum Filtrieren werden sie steif ausgestreckt und bilden unter jedem Arm zwei Filterkammreihen. Dieser Filterapparat ist sehr grob und nur in stärkeren Strömungen, die reichliches Geschwebe heranführen, wirkungsvoll.

6. Bei O. scolopendrina ist die Filtration nur für die auf trocken fallenden Habitaten wohnenden Individuen von größerer Bedeutung, bei den anderen überwiegt die Futteraufnahme vom Boden.

7. Aus den Untersuchungsergebnissen an $O$. scolopendrina muß geschlossen werden, 
daß die in größerer Tiefe am Meeresboden stellenweise massenhaft vorkommenden Opbiothrix fragilis und Ophiotbrix quinquemaculata, die dort z. T. auf dem Rücken liegen und in der Regel ihre Arme erhoben halten, nicht, wie vielfach angenommen, herabsinkende Nahrung auffangen, sondern diese ausschließlich aus stärkeren und an organischem Material reichen Strömungen abfiltrieren.

8. Der Nahrungserwerb der 20armigen, nachtaktiven und in lagunenartigen Flachwassergebieten mit schwachen Strömungen lebenden $H$. savignyi geschieht ausschließlich durch Filtration feinen, vorwiegend lebenden Geschwebes.

9. Die Arme stellen sich, die aboralen Seiten gegen die Strömung gerichtet, nebeneinander und bilden zusammen einen einheitlichen flachen Filtrationsfächer, den bei einer Strömung von $2 \mathrm{~cm} / \mathrm{sec}$ etwa $60 \mathrm{l} / \mathrm{min}$ passieren. Das feinmaschige Filternetz wird von den zwischen den Pinnulae steif ausgestreckten Tentakelreihen dargestellt.

10. Diese Befunde machen wahrscheinlich, daß auch die anderen Crinoiden, im Gegensatz zur bisherigen Anschauung, ihre Nahrung ausschließlich oder vorwiegend durch Filtration aus einer Strömung gewinnen, der nicht ein aus den oralen Armseiten gebildeter Fangtrichter, sondern die aboralen Armseiten als Filtrationsfächer entgegengestellt werden.

Die Reisen wurden mit Unterstïtzung durch die Deutsche Forschungsgemeinschaft, den Herrn Hessischen Minister für Erziehung und Volksbildung, die Vereinigung von Freunden der Technischen Hochschule zu Darmstadt, die Technische Hochschule Darmstadt und die Universität Kairo durchgeführt. Herrn Prof. Dr. H. A. F. GoHaR, dem Direktor der M.B.Station Al-Ghardaqa, und Herrn Dr. A. A. AL-Kholy, ebendort, habe ich für ihre große Hilfsbereitschaft zu danken, Herrn cand. rer. nat. L. Vö̌ker für die Mitarbeit bei den Untersuchungen 1962, Herrn Prof. Dr. G. Cherbonnier, Muséum National d'Histoire Naturelle in Paris, für die Bestimmung der Echinodermen und Frl. E. Althaus für die Ausführung der Zeichnungen.

\section{ZITIERTE LITERATUR}

BUCHANAN, J. B., 1962. A re-examination of the glandular elements in the tube feet of some common British ophiuroids. Proc. zool. Soc. Lond. 138, 645-650.

Crossland, C., 1938. The coral reefs at Ghardaqa, Red Sea. Proc. zool. Soc. Lond., Ser. A, 108, 513-523.

CzIHAK, G., 1959. Vorkommen und Lebensweise der Ophiothrix quinquemaculata in der nördlichen Adria bei Rovinj. Thalassia jugosl. 1 (6-10), 19-27.

- \& ZEr, M., 1960. Photography, television and the use of the bottom-sampler, compared as methods for quantitative analyses of benthic populations. Rapp. Proc.-verb. Réunion C.I.E.S.M.M. 15 (2), 81-83.

EMERY, K. O., 1952. Submarine photography with the Benthograph. Sci. Mon., N. Y. 75, $3-11$.

FonTAINE, A. R., 1963. A comparative study of the integumentary mucous cells of ophiuroids. Proc. XVI int. Congr. Zool. Washington D. C. $19631,87$.

Hyman, L. H., 1955. The Invertebrates. Vol. 4. Echinodermata, McGraw-Hill, New York, London, $763 \mathrm{pp}$.

KaEsTNER, A., 1963. Lehrbuch der Speziellen Zoologie. T. 1. Wirbellose. G. Fischer, Stuttgart, $1181-1212,1341-1367$. 
MAGNus, D. B. E., 1963a. Über das "Abweiden" der Flutwasseroberfläche durch den Schlangenstern Opbiocoma scolopendrina (LAMARCK). (Verh. dtsch. zool. Ges. Wien, 1962.) Zool. Anz. (Suppl. Bd) 26, 471-481.

- 1963b. Der Federstern Heterometra savignyi am Roten Meer. Natur Mus., Frankf. 93, 355-368.

- \& VöLKer, L. Zur OKkologie und Ethologie des Schlangensterns Opbiocoma scolopendrina (LAMARCK) in der Gezeitenzone des Roten Meeres. Zool. Jb. (in Vorbereitung).

Menzies, R. J., 1963. Gencral results of biological investigations on the deep-sea fauna made on the U.S.N.S. Eltainin (U.S.A.R.P.) during cruise I between Panama and Valparaiso Chile in 1962. Int. Rev. Hydrobiol. 48, 185-200.

Nrchols, D., 1960. The histology and activities of the tube-feet of Antedon bifida. Quart.J. micr. Sci. 101, 105-117.

RIEDL, R., 1956. Automatische Photographie yon Meeresböden für ökologisch-faunistische Zwecke. Óst. zool. Z. 6, 532-541.

- 1963. Probleme und Methoden der Erforschung des litoralen Benthos. (Verb. dtsch. zool. Ges. Wien, 1962) Zool. Anz. (Suppl. Bd) 26, 505-567.

Roushdy, H. M. \& Hansen, V. K., 1960. Ophiuroids feeding on phytoplancton. Nature, Lond. $188,517-518$.

SмIтH, J. E., 1937. The structure and function of the tube feet in certain echinoderms. J. Mar. biol. Ass, U. K. 22, 345-357.

VEVERs, H. G., 1952. A photographic survey of certain areas of sea floor near Plymouth. J. Mar. biol. Ass. U. K. 31, 215-222.

\section{Diskussion im Anschluß an den Vortrag Magnus}

Ockelmann: In Verbindung mit den Ausführungen von Herrn Professor Magnus möchte ich auf die Arbeit von HANSEN \& Roushdy (1960, Nature, Lond.) hinweisen. Versuche mit Flagellaten mit $\mathrm{P}^{32}$ an Opbiocomina nigra, Opbinopholis aculeata und Ophiotbrix fragilis haben gezeigt, daß die erstgenannte Art überhaupt nicht, die zweite zu einem geringen Grad und die dritte Art sehr effektiv diese Flagellaten aus einer Suspension filtrieren. Soweit ich mich erinnere, wurde das Wasser während der Versuche durch Durchlüftung in Bewegung gehalten.

Magnus: Ich kenne diese Arbeit. Die Autoren weisen damit allein die Tatsache einer unterschiedlich starken Aufnahme des Planktons durch die betreffenden Ophiuren-Arten nad. Id vermisse darin jeden Hinweis darauf, wie sie sich den Modus der Aufnahme selbst vorstellen. Wie kann man sich die Unterschiede erklären? Liegt das an der artspezifisch verschieden starken Klebfähigkeit des Drüsenapparates der Ambulacralfüßchen? Oder findet erst sekundär eine aktive Selektion des zunächst wahllos aus dem Wasser abfiltrierten Partikelmaterials statt und wo? Man müßte das doch einmal bei direkter Beobachtung der Tiere untersuchen. 